\title{
ASOCIACIONISMO MÉDICO FARMACÉUTICO EN LA ESPAÑA DE LA SEGUNDA MITAD DEL S. XIX
}

\author{
Poder Arroyo Medina \\ Depto. Historia Contemporánea - Facultad de Geografía e Historia \\ Universidad Complutense de Madrid
}

\section{RESUMEN}

A mediados del XIX se registra en España un aumento del asociacionismo médico y farmacéutico. La actitud no es nueva, pero ahora posee un significado diferente.

Las nuevas corporaciones sanitarias se desarrollarán en torno a un concepto claramente burgués, el de la profesionalidad de sus miembros. Su finalidad será la de articular los intereses de estas clases. Se convierten en un elemento más del desarrollo del Estado liberal, individualizadas por sus fines internos que nacen de la problemática de dichas clases, tanto a nivel profesional como científico.

\section{SUMMARY}

It was happened a increase of the medical and pharmaceutical association, in the middle of XIX Century. This policy nas not new, but now it has an different significance.

The new sanitary corporation will be developed around a bourgeoisie. Dea: the profesional attitude of its members. And its aim will be articulate the affairs of these class.

They become one off the elements of the Liberal State. They are identified with theirs internal aims, that speak about the profesional and scientific problems of these class.

Las más de 150 asociaciones, corporaciones o sociedades de las que se tiene noticia existieron en la segunda mitad del siglo XIX, permiten plantear el estudio del asociacionismo sanitario español decimonónico como un proceso con identidad propia y, a la vez, como un elemento más insertable en la elaboración de un modelo de organización social más general, cuya estructura depende en buena medida del desarrollo de este tipo de formaciones.

Dicho estudio ha sido abordado hasta ahora de forma puntual en sus elementos más importantes. La única visión en conjunto realizada se debe al trabajo de Alba- 
rracín Teulon, A., centrado en las asociaciones médicas del siglo XIX, en el que se da una vsión cronológica del proceso ${ }^{1}$.

Desde la perspectiva actual la sociedad moderna parece ir definiéndose, entre otros términos, como una sociedad organizada a través de criterios múltiples y formaciones sociales complejas dirigidas por unos fines y objetivos, y vertebradas racional y burocráticamente ${ }^{2}$. Tal planteamiento tiene su punto de partida sin embargo en los cambios sociales, económicos o políticos que se irán gestando durante el siglo XIX, como consecuencia de las nuevas condiciones históricas, la industrialización, la urbanización, que llevarán a una incipiente "sociedad de masas", y en consecuencia la creciente diversificación de intereses grupales e individuales que se inscriben en una nueva cultura que gira en torno a la tecnificación y a la idea de progreso. Condiciones todas ellas que acabarán por definir la necesidad de plantear nuevos tipos de relaciones sociales.

Para el análisis del tema que nos ocupa partimos de un hecho clave: esta forma de actuación corporativa lo que supone es, en definitiva, la articulación de unos intereses particulares a través de las organizaciones que se crean y que se convierten paulatinamente en actores sociales, originando a su vez nuevas relaciones o, al menos, modificando las existentes.

Esos intereses serán los de unos grupos de profesionales definidos por un marcado papel social, potenciado aún más con el desarrollo del conocimiento científico que tendrá lugar sobre todo desde mediados del XIX; lo que les hace poseedores de unas responsabilidades y valores que les legitiman ante la sociedad en la que actúan.

El asociacionismo sanitario es un hecho indiscutible durante la segunda mitad del siglo XIX, como lo prueba el gran número de asociaciones creadas; pero si bien es cierto que este siglo supone un punto de inflexión en estas formas de organización, tampoco podemos olvidar que esas profesiones, y particularmente la médica y la farmacéutica, han contado desde antiguo con una vasta e importante tradición grupal, que sirve de referencia pero con la que a la vez se marcan importantes diferencias.

Por tanto, el asociacionismo sanitario no es un hecho aislado u original de este momento, pero es ahora cuando adquirirá su grado de desarrollo más amplio y sus rasgos más destacados.

No es tampoco un hecho exclusivamente español: son numerosos los ejemplos que siguiendo modelos compartidos surgen en otros paises, como el caso de la Sociedad Francesa de Higiene, o la también francesa Sociedad de Medicina pública e Higiene profesional, la Real Sociedad de Higiene Italiana, o la Academia de Medicina de Nueva York, las mejicanas Academia Médico Quirúrgica de Larrey o la Socie-

1 Albarracín Teulón, A. (1971) "Las Asociaciones Médicas en España durante el siglo XIX”. Cuadernos de historia de la Medicina Española, vol. X. pp. 119-186.

2 MAINTZ, R. (1987), Sociología de la Organización, Madrid, Alianza Universal, p. 11. 
dad Médico Farmacéutica de Mérida, la Sociedad de Farmacia de Argentina, la de Lima o la de Santiago de Chile; algunas de las cuales sirvieron como modelos a seguir. De esta forma entendido, el fenómeno en España sería un componente más de una realidad más general y amplia. Muchas de estas asociaciones extranjeras se mueven en torno a unos objetivos fácilmente asimilables a los del panorama español y en ambos casos los colectivos que las crean son los mismos; ello hace que los intentos españoles traten en ocasiones de contactar con sus homónimos extranjeros, para crear un flujo de comunicación, guiado por los intereses compartidos y las problemáticas semejantes o similares ${ }^{3}$.

Pero lo que parece cierto es que el asociacionismo fue en esta época un hecho aceptado por muchos de los observadores sociales, y se le dio un sentido especial, tal y como lo recogen algunos comentarios de la prensa profesional:

Si observamos con detenimiento el cuadro de la sociedad actual, su esencia, su forma y los caracteres todos que a nuestra vista la distinguen de otras anteriores, no podremos menos de reconocer que el germen que da la vida y la prosperidad, reside esencialmente en el principio de asociación, admitido por todas las escuelas y organizado en todos los terrenos ${ }^{4}$.

\section{RESPONSABLES SOCIALES Y AMPARO LEGAL}

Para comprender cómo se estructura el proceso analizado, debemos en primer lugar definir las características de aquéllos que lo llevan a cabo, que son las que van a delimitar los intereses que justifiquen las decisiones tomadas.

Los profesionales sanitarios que podemos ejemplarizar en los médicos y farmacéuticos, son miembros de un mundo pluriforme, diversificado, de contornos impre$\operatorname{cisos}^{5}$, cuyos criterios de pertenencia no pueden descansar exclusivamente en razones económicas, las cuales a menudo los acercarían más a la realidad de las clases bajas; sino que dependen más de una visión propia sobre su papel en la sociedad, de una concepción pequeñoburguesa en su forma de reaccionar y en sus objetivos inmediatos, que se matiza aún más por tratarse de grupo de personas instruidas, por ser

\footnotetext{
3 Una problemática que queda definida en sus rasgos principales en lo publicado en un semanario español en 1880, en torno al Congreso de Ciencias Farmacéuticas de Bélgica, en el que los temas propuestos fueron: la revisión de la ley sobre el arte de curar, la represión del charlatanismo, el control de la expendeduría de remedios farmacéuticos, los medios para engrandecer la dignidad de la profesión y los temas referidos a las ciencias propias de esta facultad. Semanario farmacéutico, 24-10-1880, año 9, núm. 4, pp. 25-6.

4 La Clínica, 5-1-1865, núm. 29, 2. época, Madrid, p. 476.

5 Jover ZAMORA, J. M. (1976), Política, diplomacia y humanismo popular, Ed. Turner, Madrid, p. 239.
} 
profesiones liberales, poseedoras de una propiedad intransferible, su formación y la responsabilidad que ésta conlleva, mientras España responde a un modelo de país preindustrial, en el que el papel de la burguesía y la propia burguesía es todavía socialmente débil ${ }^{6}$.

Como dijimos, las profesiones sanitarias poseían un pasado corporativo, representado a menudo por agrupaciones de tipo gremial, cofradías o montepíos ${ }^{7}$, pero ahora adoptan un elemento típicamente burgués que las caracterizará de forma definitiva: su profesionalidad; una profesionalidad que las impulsa a obtener una autonomía, reflejada en el ejercicio libre de su profesión y en un autocontrol ${ }^{8}$, que favorece necesariamente la actuación colegiada, como forma de su articulación interna9.

Pero el camino que llevó a la colegiación obligatoria en la última década del siglo, está repleto de intentos variados de unión. Son esos intentos, algunos de los cuales tuvieron un notable éxito, los que constituyen el centenar largo de ejemplos de sociedades o agrupaciones de los que nos ocupamos, y son los que configuran en su éxito o fracaso el proceso analizado.

Pero junto a cuestiones de tipo sociológico, junto a los cambios sociales planteados en la España liberal, el asociacionismo como hecho general y en consecuencia la representación sanitaria del mismo, tuvo que apoyarse en un armazón legal, que legitimará, ante las autoridades y ante la sociedad en conjunto, su propia existencia.

Es decir, este tipo de actuaciones se producen porque cuentan con el amparo de una ley que al tiempo que las regula, las va dando forma externa. Este respaldo proviene a la vez del propio proceso de consolidación del derecho de asociación.

Asociaciones de diverso tipo existieron ya desde el principio del siglo, pero esta existencia estaba más relacionada con una actitud benevolente de las autoridades, que con una regulación concreta. Esa normativa tardaría en incorporarse, y lo haría a raíz de los primeros movimientos democráticos, que en España comenzaron a partir de 1868, siendo entonces en el seno de la Revolución de Septiembre cuando se reconoce por primera vez el mencionado derecho; será la Constitución del 69 la que reafirme su carácter inherente al individuo. Ni en este texto legal, ni en el de la I República, se ponen apenas cortapisas a este tipo de actuación, salvo que el objetivo de estas corporaciones atacase la moral pública o la seguridad del Estado; sin embargo,

6 JOVER ZAMORA, J. M. (1976), p. 247.

7 FOLCH JOU, G., y PUERTO SARMiento, F. J. (1984), "Origen y evolución de las corporaciones farmacéuticas españolas", Atti e Memorie della Accademia Italiana Di Storia della Farmacia, Estratto dal N2 Agosto.

Romeu DE ARMAS, A. (1987), Historia de la Previsión Social en España, Ed. "El Albar", Barcerlona 1981, pp. 475-8.

8 FRIEDSON, E. (1978), La profesión médica, Barcelona (1. a ed. Nueva York 1970), Península, p. 83.

9 VILLACORTA BAÑOS, F., Profesionales y burócratas. Estado y poder corporativo en la España del siglo XX, 1890-1923, Siglo Veintiuno, Madrid 1989, pp. 18-9. 
después de la I República el derecho queda en suspensión y algunas asociaciones se encontrarán en dificultades.

Será finalmente la Constitución de 1876 la que haga prevalecer el derecho de asociación, aunque cincelado por leyes posteriores, entre las que destaca la de 1887, cuyo resultado final es un control administrativo importante, al tiempo que ofrece garantías de que dicho control estará regulado por las normas ${ }^{10}$.

Si es importante destacar estas cuestiones legales, es porque las agrupaciones estudiadas cumplen con las reglas en ellas establecidas, entrando dentro de la dinámica organizativa del Estado Liberal; lo que las vincula todavía más a un modelo de organización que pretende ser representativo ante la sociedad y ante los poderes públicos que la regulan. Quizá una prueba de esta correlación se halle en el hecho de que desde mediados de la década de los 70 , el número de noticias recogidas sobre nuevas asociaciones o corporaciones aumenta notablemente; la Restauración parece ser el momento propicio para que maduren unos proyectos que verán su exponente máximo en la colegiación obligatoria.

\section{LOS MOTIVOS PARA EL ASOCIACIONISMO}

Si bien es cierto que el proceso del asociacionismo sanitario español respondía a un plan general, cuando se aborda de cerca el fenómeno se encuentran los elementos que lo distinguen del contexto común en el que se inserta.

Existen una serie de razones, repetidamente aludidas por los responsables de los intentos de unión, que justifican su necesidad y los individualizan. Dichas motivaciones pueden encuadrase en dos grandes áreas, que no se excluyen entre sí, sino que al contrario se complementan y discurren en la mayoría de los casos en paralelo.

La primera de ellas se deriva del destacado desarrollo de las cuestiones sobre ciencia y técnica. Pero quizá más interesantes para un análisis social del proceso sean las otras razones que se enlazan directamente con la práctica profesional, y que constituyen una constante denuncia de la problemática de estas ocupaciones. La propia prensa profesional, testigo directo e implicado en esta problemática ofrece continuas pruebas de este acontecer:

El importante ejercicio de la farmacia en botica pública no es mirado por lo general con el debido aprecio. En tal estado se halla que muchos de sus profesores sólo difícilmente pueden atender a su subsistencia y a la de sus familias. Los derechos, los sacrificios, la moralidad, el buen sentido y la importancia de la función social de los farmacéuticos se hallan de-

\footnotetext{
10 OLíAS DE LA LiMA GETE, B. (1977), La libertad de asociación en España (1868-1974), Madrid, Instituto de Estudios Administrativos.
} 
satendidas, no sólo por la preocupación de una parte del público; sino también por las leyes. En esta situación es urgente que la clase farmacéutica proscriba toda apatía, condene toda debilidad, constituya en una opinión precisa, clara y enérgica y se presente ante el país y el gobierno con unidad de pensamiento, con aspiraciones unánimes y con decisión a fin de corregir el malestar que la perturba ${ }^{11}$.

En este texto, además de la mención a la problemática de la profesión farmacéutica, destacan dos ideas. La primera es la llamada a los farmacéuticos, una llamada que se repetirá durante todo el tiempo analizado, lo que demuestra la dificultad para encontrar soluciones a esa problemática profesional.

Por otro lado introduce un elemento importante a la hora de analizar el papel del asociacionismo: dicho elemento es el Gobierno, es decir el poder establecido. Las asociaciones cumplen casi siempre con una labor mediadora entre los grupos a los que representan y el poder de la administración dentro de los cauces del Estado Liberal. El fin de sus acciones es el de articular el juego de intereses que afecta a ambas partes. Una labor que alcanzará un punto esencial cuando en 1898 se decrete la Colegiación obligatoria ${ }^{12}$.

Respecto a las denuncias sobre la situación que se trata de mejorar, éstas pueden englobarse en una serie de problemas generales, que reaparecen una y otra vez a través de casos concretos.

El primer tema importante es la denuncia de una legislación que no se amolda a las necesidades de estas profesiones, una legislación considerada restrictiva que hace dependientes de intereses ajenos a las clases médicas, que como profesiones buscan un ejercicio liberal de su tarea, en un marco más amplio al controlado por esas leyes ${ }^{13}$.

Como profesiones al mismo tiempo, tratan en todo momento de defender el ámbito propio de su trabajo; con ello, muchas de las actuaciones del asociacionismo van encaminadas a mantener y definir un monopolio, ya sea el de los servicios médicos, o el de la elaboración y venta de medicamentos. Por ello se lucha contra los ejemplos de intrusismo y charlatanismo que suplantan a unos profesionales que tratan de demarcar claramente su papel. También se denuncia el uso de remedios extranjeros y

11 Revista Farmacéutica Española, 15-1-1861, núm. 25, año 2, p. 3.

12 La colegiación desenvuelve su campo de acción, más bien, allí donde la reglamentación jurídica del Estado y su función tutelar sobre el ejercicio profesional no alcanza, o allí donde ha perdido su capacidad de informar la realidad a que hace referencia, por razones de diversa índola, pero que se suele resumir en un problema de inadecuación de la norma a realidades nuevas del ejercicio profesional. VILLACORTA BAÑOS, F. (1989), p. 16.

13 "El farmacéutico se halla, pues, limitado por restricciones gubernativas especiales, exclusivas, sólo dirigidas hacia él, hecho del que se deriva en su mayor parte el malestar de la clase. El Estado, sin embargo, no ofrece al profesor que ejerce la farmacia en botica pública garantías bastantes para la seguridad de su subsistencia". Revista Farmacéutica Española, 15-1-1861, núm. 25, año 2, po. 3-5. 
secretos, lo que conlleva en muchos casos la crítica a farmacéuticos o médicos que realizan estas prácticas ${ }^{14}$.

En esa definición de su marco de actuación, se busca asimismo la regulación de los mecanismos de control y de los cargos administrativos encargados de ello, como las subdelegaciones a las que a menudo se acusa de no realizar correctamente su trabajo.

Por último se plantea la necesidad de convencer tanto a médicos como farmacéuticos de los beneficios que aportaría una postura de unión, evitando las rivalidades internas que debilitan la obtención de una posición más firme en el espectro social de las clases medias, lo que les dará mayor capacidad para defender sus propios intereses y derechos. A este respecto es interesante comprender que muchas de las agrupaciones que se funden serán el resultado de la cooperación entre miembros de profesiones diferentes, que comparten la idea de una clase única con intereses similares y problemas análogos. Esto no significa una identificación entre ellas: cada grupo profesional posee una cierta autonomía que se traduce en el deseo de establecer una ética propia, una deontología desde la que se regulen las relaciones entre los diversos miembros y colectivos ${ }^{15}$.

El análisis de estos elementos de protesta nos hace ver cómo el proceso analizado viene a significar la búsqueda de una nueva ordenación social para estas profesiones, basada en una nueva conciencia de su funcion social que plantea la necesidad de un cambio en los mecanismos de poder e influencia social, dentro de unas estructuras que han variado desde los modelos del Antiguo Régimen a los esquemas del Estado Liberal.

Por tanto el interés por la ciencia y la profesión que está presente en las declaraciones de objetivos que realicen estas corporaciones, tanto en la publicación de sus estatutos y reglamentos, como en los artículos periodísticos que en su nombre o en el de alguno de sus miembros fueron publicados, es un factor que unifica todo el proceso tratado y lo distingue de otros parejos.

$\mathrm{La}$ atención por la ciencia es lógica en un contexto de desarrollo técnico como el que se da en estos años. Algunas de las agrupaciones que se fundan responderán exclusivamente a las nuevas ciencias en formación. Pero la presencia de la ciencia

\footnotetext{
14 Como el caso de intrusismo producido en Zaragoza y Logroño, con motivo de la comercialización de los productos de un extranjero conocido como Sequah y que provocó una notable reacción de los farmacéuticos afectados, que hicieron llegar sus quejas hasta el Parlamento por mediación del diputado vallisoletano Sr. Muro, dirigiéndose en sus reclamaciones al ministro de la Gobernación: en él se acusaba de negligencia y complicidad a los poderes públicos del Gobernador de Zaragoza y de Logroño. Farmacia Moderna, 5-2-1892, núm. 4, p. 66; 15-3-1892, núm. 9, pp. 144-5; 5-4-1892, núm. 10, pp. 159-62.

15 Aparecen ahora obras sobre estos temas de moral y deontología como: JANER Y BERTRÁN (1831), Elementos de Moral Médica, Barcelona; o Simón MARX, (1832), Deontología Médica, traducido por Francisco Ramos, citado por HIGUERAS LÓPEZ, E. (1982), Medicina y ética en la España del XIX, Tesina leída en UCM.
} 
puede ser entendida a su vez como una herencia ilustrada, derivada de las Academias de Ciencias y Artes y de los Laboratorios científicos que habían proliferado un siglo antes.

Pero desde 1845, con la Reforma de la Universidad, la investigacion científica pasó a ser labor central de esta institución, lo que disminuía la capacidad de las organizaciones particulares para realizar un trabajo científico práctico, por lo que en la mayoría de los casos el interés en esta materia de las asociaciones o corporaciones quedó reducido a debates sobre cuestiones más o menos modernas, o análisis de las nuevas ideas importadas desde los países más avanzados y sobre todo la emisión de informes y dictámenes en torno a temas variados ${ }^{16}$. Este desplazamiento de la temática científica irá en aumento según transcurran las décadas y, a finales del siglo, se fundarán corporaciones cuyo único centro de acción es el campo profesional.

Quizá sea ésta una prueba más de la importancia que el componente de lo social tuvo para estos colectivos, ya que esa problemática encierra un deseo de participar e incluso crear nuevas vinculaciones sociales, en las que los colectivos sanitarios van convirtiéndose en la medida de lo posible en grupos de interés, tratando de adquirir los instrumentos y la fuerza necesaria para ser grupos de presión, y poder influir en las decisiones de aquéllos que pueden modificar su situación, es decir en el Gobierno y en la elaboración del marco jurídico que les afecte, a la vez que definen su posicion en el propio mercado laboral a través de su actividad monopolística.

\section{MODELOS DE ASOCIACIÓN}

La fundación de las corporaciones sanitarias se llevó a cabo siguiendo modelos diversos, bajo denominaciones diferentes que en algunos casos definían externamente el contenido interno de las mismas. En función de sus fines y objetivos, que determinan de forma general las actividades de estas corporaciones, pueden ser clasificadas en tres tipos: aquellas con un doble interés, tanto en asuntos científicos como en profesionales, las que sólo manifiestan unos fines científicos y las que centran sus acciones a nivel exclusivamente profesional.

Dentro de esta división, se establece a su vez una destacada diversidad de modelos: colegios profesionales, institutos, asociaciones, sociedades, academias, ateneos, centros, etc.

\footnotetext{
16 Informe del Colegio de Farmacéuticos de Granada sobre la obra "Enciclopedia Farmacéutica", Semanario Farmacéutico, 21-4-1889, núm. 29, p. 247. La Academia de Ciencias Médicas de Badajoz, en su Sección de Higiene, se encargó de publicar un folleto titulado "Limpieza de la población", Semanario Farmacéutico, 14-10-1883, núm. 2, p. 16.
} 
El estudio de los diversos tipos organizativos, en sus exponentes más importantes o conocidos, permite comprobar la existencia de elementos recurrentes o compartidos en todas ellas, muchos de los cuales se explican por el hecho de que seguían modelos ya establecidos y que actuaron en todo el período como guías para las nuevas creaciones. La forma de unión que a este respecto mostró siempre mayor capacidad de desarrollo fue la colegial, una de las primeras en instituirse, y la que alcanzó mayor éxito cuando a finales de siglo se convierta en oficial la colegiación obligatoria; pero antes de esto los intentos fueron muchos y singulares.

\section{Academias y ateneos}

Ambos tipos de agrupación no son muy numerosos, y responden de una forma mayoritaria a agrupaciones científicas, entendiéndolas como centros de debate en los que los temas y cuestiones científicas eran expuestos a análisis a través de jornadas de conferencias y de estudio. En muchos casos se planteó la posibilidad de crear gabinetes o laboratorios en los que llevar a la práctica algunas de estas teorías, pero la realidad fue que estas agrupaciones no contaron con los medios materiales necesarios para ello, mientras que la investigación quedaba restringida a otros organismos.

Respecto a las academias, podemos mencionar la Academia de Ciencias Médicas de Badajoz (1877), la Academia Médico Farmacéutica (1876) y la Academia y Laboratorio de Ciencias Médicas de Cataluña (1878): son un tipo de agrupación provincial que posiblemente surgieron a la sombra de las grandes Academias de Medicina o Farmacia de Madrid o Barcelona, uno de los vestigios heredados del pasado ilustrado.

Sus objetivos eran simples y no disentían de lo que será la tónica general: reunir en un centro común las inteligencias y actividad de todos los profesores de Ciencias Médicas... para procurar el adelantamiento de las mismas y el decoro y el bienestar de los que las profe$\operatorname{san}^{17}$.

\section{Centros}

Su número es escaso; existen noticias sobre el Centro Médico castellonense "La Unión" (1872), el Centro Médico Farmacéutico de Manresa (1878) y el Centro Médico Farmacéutico de Tarragona (1887). Y sus objetivos suelen moverse en la línea general recogida también por el anterior grupo. Alguna diferencia la muestra el ejemplo mallorquín, ya que entre sus motivaciones estaba la de ser un centro distri-

17 Estatutos y Reglamento de la Academia Provincial de Ciencias Médicas de Badajoz (1877), Badajoz, Imp. José Santamaría y Navaro. 
buidor y elaborador de drogas y medicamentos a gran escala, oponiéndose con ello a la actividad de las droguerías.

\section{Sociedades}

En este caso la multiplicidad de intereses representados por estas corporaciones es mayor, existen sociedades a las que vinculan únicamente intereses científicos, como la Sociedad de Terapéutica y Farmacología (1880), en cuyos estatutos se alude a un único fin en el desarrollo de esta rama de la Medicina, en pleno auge por estas décadas.

Pero quizá una de los ejemplos más interesantes, no sólo en este grupo sino en todo el proceso asociacionista general, lo constituye la Sociedad Española de Higiene (1881). Y es interesante por la propia temática abordada, la Higiene y la Salud pública, que cuenta a finales del siglo con un protagonismo propio, con repercusiones no sólo científicas sino también sociales. Además, este tipo de agrupaciones es frecuente en los países occidentales; su presencia en España viene a significar el intento de unos grupos de profesionales y hombres de ciencia por situar a este país en el ámbito de los más desarrollados. Y finalmente esta sociedad es destacable por el apoyo oficial con el que contó desde su creación, avalada por la presencia en su sesión inaugural de Alfonso XII y porque incluyó entre sus fundadores y cargos a personajes de la talla de Carlos María Cortezo y Francisco Méndez Álvaro.

Su finalidad era estudiar la forma de:

Vivir más y vivir mejor; acrecentar el bienestar físico; ayudar al desarrollo de las facultades individuales; velar por la protección del débil; acudir al que comienza a vivir; aconsejar al ignorante; reprimir al desaconsejado; atraer la atención de las colectividades y la actividad del Estado sobre puntos si no ignorados, olvidados al menos en medio de los vaivenes de nuestra accidentada vida política; estos y muchos otros problemas se imponían con el carácter de cuestiones de interesante estudio, al propio tiempo que con el de la necesidad imperiosa ante la naciente sociedad ${ }^{18}$.

Tales propuestas parecen preludiar un cambio en la concepción social de las ciencias sanitarias, como resultado del cambio real que comienza a manifestarse dentro de una sociedad que requiere nuevas soluciones para problemas nuevos ${ }^{19}$.

\footnotetext{
18 Discursos de la Solemne inauguración de la Sociedad Española de Higiene (1882), Madrid, Impr. Enrique Teodoro, calle de Atocha.

19 España es, a este respecto, reflejo tardío y más difuminado de las ideas que han ido fructificando desde la década de los 40 en Alemania bajo la labor de médicos como Virchow o Neumann, que convertían el problema médico en un problema social en el sentido más amplio del término, y que en conse-
} 
Otros ejemplos de sociedades menos relevantes se acercan al papel desarrollado por academias o ateneos; es el caso de la Sociedad Farmaco-Científica. Hubo también agrupaciones en las que sus miembros eran no profesores médicos, sino los alumnos de las respectivas facultades: fueron la Sociedad Farmacéutica Escolar o la Sociedad Escolar "La Juventud Farmacéutica", que si bien no tuvieron un papel destacado en el contexto general del que partimos, lo que vienen a significar es el nivel de aceptación del asociacionismo, a la vez que pueden reiterar el peso alcanzado por la Universidad como centro aglutinador del interés científico, siendo alguno de sus cargos los profesores más notables de estas facultades.

Dentro de este grupo encontramos también sociedades puramente profesionales. que a su vez muestran gran variedad de intereses. Hay sociedades de naturaleza mercantil como la Sociedad Farmacéutica Española (1881), sociedad fundada con carácter colectivo en la forma de sociedad comandita por acciones, bajo la razón de G. Formiguera y Compañía, y que pretendía contribuir al control del mercado farmacéutico actuando como centro distribuidor de productos y artículos farmacéuticos de procedencia española, lo que supone una forma de luchar contra la competencia extranjera.

Junto al aspecto claramente monopolizador de estas organizaciones, existe también otra perspectiva que afecta a un hecho comprobable, la numerosa comercialización de sustancias medicamentosas y de establecimientos expendedores de éstas, ajenas o paralelas al menos a la oficina de farmacia, cuyo mal no reside en su abundancia sino en la posible falta de rigor y de respaldo científico de las mismas.

Pero además el planteamiento de cuestiones de este tipo nos pueden abrir nuevos campos de análisis, en torno a la producción farmacéutica que en estos momentos se mantiene muy unida a un modelo tradicional, por el cual los medicamentos se realizaban mayoritariamente en los laboratorios de las boticas, una forma artesanal frente a otras posibilidades industriales. Por ello la petición de laboratorios centrales y almacenes de estos productos quizá conlleve nuevas concepciones a nivel productivo.

Entre los intereses de la Sociedad Farmacéutica Española estuvo también la creación de un Montepío de ayuda a los profesionales y a sus familias. La existencia de sociedades mutualistas corresponde a otra modalidad de unión, más restringida en sus funciones u objetivos. Entre las creadas en estos momentos están la Sociedad Filantrópica de Profesores de Ciencias Médicas (1851), el Montepío Facultativo (1858), la proyectada Sociedad de Seguros "El porvenir del Farmacéutico" (1864),

cuencia exigía para su resolución, no sólo el avance técnico de la ciencia de curar, sino el propio desarrollo del pensamiento político, cuyo resultado serían las necesarias y profundas reformas políticas, enclavadas en los anhelos de la Revolución del 48. ROSEN, George (1985), De la Policía médica a la Medicina social, México (1. ${ }^{a}$ ed., en inglés, 1974), Siglo XXI, pp. 77-88. 
contra la que hubo reticencias y oposiciones ${ }^{20}$, o la Sociedad Balnearia Médico Farmacéutica Española (1890).

En su mayoría son intentos parciales pero que responden a una necesidad social concreta, la falta de seguridad para unos grupos que sobreviven en relación a su propio trabajo, y la precariedad económica a la que se ven sometidos estos grupos en aquellas circunstancias en las que el facultativo responsable viese mermada o anulada su capacidad laboral; esto permite comprender cómo estas profesiones, en principio insertas dentro de las clases medias, responden a menudo a realidades más cercanas a las clases bajas o populares. Lo que viene a complicar el panorama real de la sociedad española de la época.

Por último podemos encontrar algun caso de sociedad con intereses mixtos, tanto profesionales como científicos, pero son muy escasas como el caso de la Sociedad de Farmacia de Valladolid (1883).

\section{Asociaciones}

Los objetivos de este grupo suelen ser mixtos o profesionales, como en la Asociación Médico Farmacéutica Española que, fundada en 1872, desparecería dos años después, y que fue planteada como un medio de comunicarse, entenderse, respetarse y fortalecerse en la unión del compañerismo, en el exacto cumplimiento de sus deberes, el recto juicio de sus derechos... En esta agrupación aparece ya un tema importante, la conveniencia de la colegiación médica obligatoria ${ }^{21}$.

Más tarde, bajo el nombre de Asociaciones Médico Farmacéuticas se fundarán en la última década del siglo numerosas agrupaciones provinciales o locales, que supusieron un renacer espontáneo del proceso corporativista encaminado directamente ya a la consecución de la colegiación obligatoria.

Otras, como la Asociación Farmaceûtica Matritense (1878), giraron una vez más en torno a la problemática de la clase a la que representar.

También las hubo que aceptaron una labor mixta, la Asociación Farmacéutica Española (1867), la Asociación de San Sebastián (1877), la Asociación Asturiana de las Clases Médicas (1883) o la Asociación Médica Farmacéutica Veterinaria Provincial (1884). Pero en este panorama destaca de una forma especial la Asociación de la Prensa Médico Farmacéutica (18775), que además de su directa vinculación con las

20 Semanario Farmacéutico, 31-7-1887, núm. 44, año 17, p. 351.

21 Semanario Farmacéutico, 27-4-1873, núm. 30, año 1. 
profesiones médicas, significó uno de los primeros ejemplos del asociacionismo periodístico en España ${ }^{22}$.

Su visión sobre la situación de estas clases es clara: si de la misma manera que hoy se encuentra unida la prensa médico-farmacéutica, lo estuviera la clase toda, no dudaríamos ni por un momento que ésta conseguiría lo que la justicia reclama y lo que sus irremplazables servicios a la sociedad le hacen acreedora ${ }^{23}$.

A la Asociación de la Prensa se suscribieron la casi totalidad de los periódicos médicos y farmacéuticos madrileños y su influencia pronto se dejó sentir en los del resto del país. En 1883 el número de adhesiones había crecido considerablemente

\section{PERIÓdICOS PRESENTES}

El Siglo Médico

El Genio Médico Quirúrgico

La Correspondencia Médica

La Farmacia Española

El Jurado Médico Farmacéutico

Los Avisos

La Gaceta Médico Veterinaria

El Semanario Farmacéutico

La Revista de Medicina Dosimétrica

El Boletín Clínico del Instituto

Homeopático

Los Anales de la Sociedad Española de Hidrología

La Oftalmología Práctica

La Higiene

Los Anales de Cirugía

La Revista de Medicina y Cirugía

Prácticas

La Medicina Rural

Los Anales de Obstetricia

La Revista de Enfermedades de los Niños

La Gaceta Médica de Sevilla ${ }^{24}$

\section{PERIÓDICOS ADHERIDOS}

La Unión Médica (Ávila)

La Hidroterapia

La Clínica (Zaragoza)

La Revista Extremeña de Medicina, Cirugía y Farmacia (Cáceres)

El Restaurador Farmacéutico (Barcelona)

La Gaceta Médico Catalana (Barcelona)

La Revista de Ciencias Médicas (Barcelona)

La Enciclopedia Médico Farmacéutica (Barcelona)

El Monitor de la Salud (Barcelona)

La Revista de Higiene (Barcelona)

La Academia Molinesa (Guadalajara)

La Gaceta de los Hospitales (Valencia)

Boletín del I. Médico Valenciano (Valencia)

La Crónica Médica (Valencia)

Revista Médico Farmacéutica (Castellón)

Revista Médica Vasco-Navarra (Vitoria)

La Guía de la Salud (Sevilla)

La Revista Médica de Sevilla (Sevilla)

La Prensa Médica de Granada (Granada)

La Gaceta de Higiene y Climatología (Cádiz)

22 Fernández SANZ, J. J. (1992), "La Asociación de la Prensa Médico Farmacéutica (1875), pionera del asociacionismo periodístico español", Asclepio, II, pp. 43-4.

23 Semanario Farmacéutico, 26-12-1875, núm. 13, p. 121.

24 Semanario Farmacéutico, 26-12-1875, núm. 27, año 11, p. 225. 
También algunas asociaciones de partido se sumaron en su apoyo.

La relación entre prensa profesional y asociacionismo sanitario es directa, ya que muchas de estas publicaciones fueron los órganos oficiales de las organizaciones. Pero sobre todo porque fueron casi siempre vehículo de las ideas y anhelos de las clases a las que se dirigían, sufriendo un proceso similar al del corporativismo, con la fundación de numerosos intentos periodísticos, muchos de los cuales desaparecieron con la misma rapidez con la que se formaron.

\section{Institutos}

Dos son los ejemplos más importantes; el Instituto Médico Valenciano (1841) y el Instituto Farmacéutico Aragonés (1851), que si bien muestran diferencias importantes, sobre todo en su desarrollo como corporaciones, más completo y destacado en el caso valenciano, también poseen un perfil similar, con intereses compartidos entre el campo científico y el profesional.

Además, las dos formaciones se hallan ligadas a la creación de los respectivos colegios provinciales de médicos y farmacéuticos. De hecho la primera intención de los profesores aragoneses fue la de fundar un colegio, lo que no fue posible por la existencia de un contencioso referente a un colegio anterior ${ }^{25}$.

Ambos desarrollaron su actividad en los dos planos establecidos, e incluso desde dentro de éstos diversificaron sus acciones en distintas disciplinas; así, el Instituto valenciano realizó una labor social, otra higiénico-sanitaria y una más general que abordó temas científicos como los farmacológicos ${ }^{26}$. En el caso aragonés aparecen elementos propios de la profesión farmacéutica como un depósito de medicamentos que vendría a cumplir con las pretensiones de otras asociaciones en las que hemos visto la posibilidad de elaborar y distribuir medicamentos y productos farmacéuticos. A este tipo de agrupaciones se las consideraba capacitadas para realizar labores a este nivel; así, en repetidas ocasiones fueron requeridas para elaborar análisis y estudios sobre remedios secretos o extranjeros con el fin de emitir informes públicos.

La lucha contra ese mercado paralelo fue a menudo causa de unión dentro del propio movimiento asociacionista, sobre todo a nivel farmacéutico; el Instituto Farmaceútico Aragonés fue llamado para ello en varios momentos, como en 1857 cuan-

\footnotetext{
25 Actas de la Junta General del IFA (sesión del 16-9-1851). Libro 34, Archivo de la Real Academia de Farmacia.

26 TERUEl PIERA, S. (1974), Labor del Instituto Médico Valenciano, Madrid, CSIC. FRESQUET FERRER, J. L. (1985), "La farmacología y el Instituto Médico Valenciano 1841-1896", Cuadernos Valencianos de Historia de la Medicina y de la Ciencia, XXVIII, serie de monografías. Cátedra de Historia de la Medicina. Universidad de Valencia, pp. 59-70.
} 
do el Colegio de Farmacéuticos de Barcelona le invita a unirse a él para conseguir del gobierno el cumplimiento de las Leyes de Sanidad ${ }^{27}$.

El propio Instituto Aragonés trató en todo momento de contribuir a una unión general de los farmacéuticos españoles, apoyando desde el principio el Congreso de Madrid de 1865, en el que actuó como su representante Quintín Chiarlone, uno de los farmacéuticos más activos y destacados en estos años, y aprobando el Reglamento de la Asociación Farmacéutica Española, proyecto avalado en esta reunión y del que fue fiel defensor.

\section{Colegios profesionales}

Por último, se encuentran los Colegios como la gran aportación del corporativismo sanitario decimonónico, tanto a la ordenación interna de estas clases y como a la elaboración de nuevas relaciones sociales.

Estas organizaciones cuentan además con una historia propia que las individualiza dentro del contexto general, en el que sin embargo se insertarán bajo el peso de las circunstancias y de los cambios históricos. Así, el Colegio de Farmacéuticos de Madrid aprueba sus estatutos en 1737, un texto que será reformado en 1855, estableciéndose con él el modelo más recurrente dentro de esta profesión, ya que en la organización de otros Colegios provinciales, como el de Valencia, se remiten al madrileño como referente obligado, mientras que otros se estructuran sobre la base de sus criterios y sus normas. De hecho se le concedió la representatividad de todo el colectivo nacional en las ocasiones que lo requiriesen.

Los Colegios fundados así bajo la tutela del de Madrid, van a establecer unos fines mixtos, que mantienen la fórmula acostumbrada de la propagación de los adelantos científicos, el buen orden del ejercicio de la profesión y contribuir al decoro y prosperidad de la clase.

Sin embargo, centrándonos en el caso de la Farmacia, podemos distinguir dos periodos en la creación de colegios: el primero, marcado por el seguimiento de este Colegio, dará como resultado los Colegios de Sevilla (1853), Granada (1856), Barcelona (1857), Cádiz (1858), el Colegio de Farmacéuticos de Valencia (1864) y el Colegio de Farmacéuticos de Castilla la Vieja (1865), único en su género por tratarse de un colegio regional y no provincial.

La siguiente etapa surgirá a partir de los setenta; en ella empiezan a primar los intereses profesionales sobre los viejos esquemas heredados de los modelos del Antiguo Régimen que favorecían mas las propuestas científicas. En este cambio influi-

27 Acta de la Junta General del IFA (sesión del 20-7-1857), Libro 34, Archivo de la Real Academia de Farmacia, Madrid. 
rán determinados acontecimientos como la Celebración del Congreso Farmacéutico Nacional de 1865 y la actuación de Fernández Izquierdo. De esta segunda etapa son los Colegios de Santander (1878), Córdoba y Málaga (1880), Zaragoza (1882), Lérida (1892) y Zamora (1892). Esta lista se cierra con el Colegio de Farmacéuticos de Murcia, cuyos Estatutos, fechados en 1897, recogen en su segundo artículo que: $E l$ Colegio de Farmacéuticos de Murcia no se ocupará más que de asuntos profesionales o de los que con ellos tengan relación, siendo por tanto la primera corporación de este tipo que elimina de sus objetivos, al menos inicialmente, toda referencia a la propagación de la ciencia.

Los médicos también se unen pronto a este tipo de acciones y en 1854, a raíz de la creación del periódico El Siglo Médico, se hace un llamamiento a estos profesionales para retomar la vieja idea de un colegio profesional que desde Madrid actúe como ejemplo para las otras provincias que enseguida se animan, empezando por Barcelona. Pero problemas políticos paralizan este proceso, que se revitalizará de una forma general durante la década de los 90 a través de las correspondientes Asociaciones Médico Farmacéuticas provinciales ${ }^{28}$.

Surgen en estos años finales del siglo otra modalidad de Colegio, que se diferencia básicamente por ser, como esas Asociaciones provinciales, corporaciones mixtas de ambas profesiones como el Colegio Médịco Farmacéutico de Logroño (1892), el de Zaragoza (1892) y el Colegio Médico Farmacéutico Balear (1894).

Cada decisión que se toma, cada acción que se emprende en estos años, tiene un claro objetivo: conseguir el establecimiento de la colegiación obligatoria, lo que significa construir de forma definitiva una organización de estas clases, tanto a nivel interno como en sus vinculaciones con los otros grupos sociales y los poderes legítimos del nuevo Estado Liberal. Pero la obligatoriedad de la medida afecta directamente al concepto básico de libertad profesional y se inician las primeras muestras de descontento y oposición. A pesar de ello, el 12 de abril de 1898, con la firma del ministro de la Gobernación Ruiz y Capdepón en el gobierno Sagasta, se aprueban los Estatutos para el régimen de los Colegios Médicos y Farmacéuticos.

Con ello, el proceso iniciado con los primeros intentos de unión por parte de los profesores de estas facultades parece haberse cumplido; esos mismos profesores como parte integrante de la sociedad, se han incorporado a las nuevas formas de ordenación que las nuevas estructuras sociales requieren. El debate iniciado en torno a la legitimidad de la obligatoriedad o no de la colegiación, que se iniciará a partir de estos años, introduce sin duda nuevas alternativas a un resultado por otra parte ineludible en la construcción de la actual sociedad organizada.

28 Albarracín Teulón, A. (1971). 
CORPORACIONES SANITARIAS EXISTENTES ENTRE 1840-189929

$-1840-1849$

- Asociación Médica de Jerez de la Frontera (1840)

- Asociación de Médicos y Cirujanos de Ávila (1840)

- Instituto Médico Español (1840).

- Academia de Emulación de Ciencias Médicas (1841)

- Asociación Médica del Puerto de Santa María (1841)

- Asociación de Médicos y Cirujanos de Vigo (1841)

- Instituto Médico Alicantino (1841)

- Instituto Médico Murciano (1841)

- Instituto Médico Toledano (1841)

- Instituto Médico Valenciano (1841)

- Sociedad Médica de Emulación (1841)

- Academia de Esculapio (1842)

- Instituto Médico General de España (1842)

- Asociación Médica de Lugo (1843)

- Asociación Médica de Toledo (1843)

- Asociación Médica de Cartagena (1843)

- Ateneo Médico Sevillano (1843)

- Instituto Médico Guipuzcoano (1843)

- Instituto Médico de Lucena (1843)

- Instituto Médico del Puerto de Santa María (1843)

- Sociedad Médico Quirúrgica de Málaga (1843)

- Sociedad Médico Quirúrgica de Santander (1843)

- Sociedad Médico Quirúrgica de Segovia (1843)

- Academia Quirúrgica Matritense (1845)

- Sociedad de Emulación y Filantropía de los Hospitales Generales (1845)

- Sociedad Farmacéutica de Socorros Mutuos (1845)*

- Confederación Médica (1847)

29 Las fechas recogidas no tienen por qué coincidir con las de la creación de las distintas organizaciones; sólo de aquéllas de las que poseemos documentación oficial, como Estatutos o Reglamentos, o bien actas y noticias sobre su inauguración podemos afirmarlo con seguridad —son los nombres marcados con un asterisco-; el resto corresponden a las fechas de las correspondientes a las noticias halladas, que suelen ser normalmente noticias o reseñas periodísticas - la prensa es una fuente esencial en este trabajo-, o las dadas por otros investigadores. 
PODER ARROYO MEDINA

$-1850-1859$

- Sociedad Filantrópica de Profesores de Ciencias Médicas (1851)*

- Instituto Farmacéutico Aragonés (1851)*

- Colegio de Farmacéuticos de Sevila (1853)*

- Alianza de las Clases Médicas (1854)

- Emancipación Médica (1854)

- Museo Español de Medicina (1854)

- Academia de Medicina y Ciencia de Barcelona (1855)

- Círculo Médico Farmacéutico Barcelonés (1855)

- Asociación Médico Provincial de Segovia (1855)

- Unión Médica (1855)

- Asociación de Protección Médico Quirúrgica Farmacéutica Veterinaria de Daroca (1856)

- Colegio de Farmacéuticos de Granada (1856)*

- Academia Científico Literaria (1857)

- Colegio de Farmacéuticos de Cádiz (1858)*

- Monte Pío Facultativo (1859)*

- Academia Médico Quirúrgica Matritense (1859)

$-1860-1869$

- Círculo Médico (1861)

- Centro Médico (1862)

- Cuerpo de Sanidad Civil del Dr. Cuesta (1862)

- Instituto Catalán de Vacunación (1862)

- La Amiga del Estudio (1864)

- Colegio de Farmacéuticos de Valencia (1864)*

- Colegio de Farmacéuticos de Castilla la Vieja (1865)*

- Instituto Gaditano de Ciencias Médicas (1865)

- Instituto Médico (1865)

- Asamblea Valencia (1866)

- Asociación Farmacéutica Español (1867)* 
- La Joven Farmacia (1871)*

- Asociación Médico Farmacéutica Española (1872)*

- Centro Médico Castellonense de La Unión (1872)*

- Asociación Médico Farmacéutica de la Provincia de Castellón (1873)

- Ateneo Médico Farmacéutico del Hospital Nacional (1873)*

- Instituto de Vacunación del Estado (1874)*

- Colegio Médico Farmacéutico Español (1875)*

- Sociedad Española de Historia Natural (1875)*

- Asociación de la Prensa Médico Farmacéutica (1875)*

- Academia Médico Farmacéutica de Barcelona (1876)

- Ateneo Propagador de Ciencias Naturales (1876)

- Centro General de Vacunación (1876)*

- Academia Médico Farmacéutica Escolar (1877)

- Academia Médico Quirúrgica Jerezana (1876)

- Academia Provincial de Ciencias Médicas de Badajoz (1877)*

- Asociación de San Sebastián (1877)

- Sociedad Española de Hidrología Médica (1877)*

- Academia de Ciencias Médicas de Cataluña (1878)

- Academia y Laboratorio de Ciencias Médicas de Cataluña (1878)*

- Asociación Farmacéutica Matritense (1878)

- Centro Farmacéutico de Mallorca (1878)*

- Centro Médico Farmacéutico de Manresa (1878)*

- Colegio de Farmacéuticos de Santander (1878)

- Asociación Médico Farmacéutica de Cartagena (1879)

- Asociación Médico Quirúrgica de Valladolid (1879)

- Asociación de Valmaseda (1879)

- Colegio de Farmacéuticos de Tarragona (1879)*

$-1880-1889$

- Asociación Médico Farmacéutica de Albacete (1880)

- Asociación Médico Farmacéutica de Aliaga (1880)

- Asociación Médico Farmacéutica de Orense (1880)

- Colegio de Farmacéuticos de Málaga (1880)

- Instituto Médico Quirúrgico de León (1880)

- Los Escolares Médicos (1880)

- Sociedad Española de Terapéutica y Farmacología (1880*

- Sociedad de Ciencias Médicas de Las Palmas (1880) 
PODER ARROYO MEDINA

- Colegio de Farmacéuticos de La Habana (1881)*

- Asociación de Socorros Mutuos de Valladolid (1881)*

- La Juventud Farmacéutica (1881)*

- Sociedad Española de Higiene (1881)*

- Sociedad Farmacéutica Escolar (1881)

- Sociedad Médica Farmacéutica de los Santos Cosme y Damián (1881)

- Asociación Médico Quirúrgica Sepulvedana (1882)

- Sociedad Farmacéutica Española (1882)*

- Academia Médico Quirúrgica Española (1883)

- Asociación Asturiana de Clases Médicas (1883)

- Asociación Médico Farmacéutica Veterinaria Provincial (1883)

- Asociación de Fontiveros (1883)

- Asociación Médico Farmacéutica de Teruel (1883)

- Ateneo Escolar Gallego (1883)

- Sociedad Farmacéutica de Valladolid (1883)

- Sociedad de Medicina Legal (1883)

- Ateneo Médico Farmacéutico Matritense (1884)

- Sociedad Farmaco Científica (1884)

- Academia Médico Quirúrgica Farmacéutica de Las Palmas (1884)

- Ateneo de Ciencias Antropológicas (1886)

- Ateneo Farmacéutico Escolar (1886)

- Asamblea Farmacéutica Española (1887)

- Centro Médico Farmacéutico de Tarragona (1887)*

- Colegio Médico Farmacéutico de Orense (1887)

- Colegio Médico Farmacéutico de Gandía (1887)

- Ateneo Zaragozano (1888)

- Asociación Médico Farmacéutica de Mataró (1888)

- Asociación Médico Farmacéutica de Baltanas (1889)

$-1890-1900$

- Asociación Médico Farmacéutica de Rioseco (1890)

- Centro de Vacunación y Revacunación de la Provincia de Álava (1890)

- Sociedad Balnearia Médico Farmacéutica Española (1890**

- Asociación Médico Farmacéutica de Navalcarnero (1891)

- Asociación Médico Farmacéutica de Almendralejo (1892)

- Asociación Médico Farmacéutica de Badajoz (1892) 


\section{ASOCIACIONISMO MÉDICO FARMACÉUTICO EN LA ESPAÑA DE LA SEGUNDA MITAD DEL XIX}

- Asociación Médico Farmacéutica de Béjar (1892)

- Asociación Médico Farmacéutica de Burgos (1892)

- Asociación Médico Farmacéutica de Calatayud (1892)

- Asociación Médico Farmacéutica de Caspe (1892)

- Asociación Médico Farmacéutica de Ciudad Rodrigo (1892)

- Asociación Médico Farmacéutica de Chinchilla (1892)

- Asociación Médico Farmacéutica de Ejea de los Caballeros (1892)

- Asociación Médico Farmacéutica de Estella (1892)

- Asociación Médico Farmacéutica de La Guardia (1892)

- Asociación Médico Farmacéutica de Huelva (1892)

- Asociación Médico Farmacéutica de Los Infantes y de Saldaña (1892)

- Asociación Médico Farmacéutica de Játiva (1892)

- Asociación Médico Farmacéutica de Logroño (1892)

- Asociación Médico Farmacéutica de Navarra (1892)

- Asociación Médico Farmacéutica de Olmedo (1892)

- Asociación Médico Farmacéutica de Palencia (1892)

- Asociación Médico Farmacéutica de Sepúlveda (1892)

- Asociación Médico Farmacéutica de Tarazona (1892)

- Asociación Médico Farmacéutica de Tolosa (1892)

- Asociación Médico Farmacéutica de Utrera (1892)

- Asociación Médico Farmacéutica de Villalpando (1892)

- Asociación Médico Farmacéutica de Villanueva de la Serena (1892)

- Asociación Guipuzcoana (1892)

- Asociación General de Médicos y Farmacéuticos rurales (1892)

- Asociación General de Médicos y Farmacéuticos (1892)

- Colegio Médico Farmacéutico de Oviedo (1892)

- Colegio Médico Farmacéutico de Zaragoza (1892)

- Colegio de Farmacéuticos de Lérida (1892)

- Colegio de Farmacéuticos de Zamora (1892)

- Asociación Médico Farmacéutica de Tarancón (1893)

- Asociación Médico Farmacéutica de Zaragoza capital (1893)

- Colegio Médico Farmacéutico Navarro (1893)

- Colegio Médico Farmacéutico del Reino de Valencia (1893)

- Asociación Médico Farmacéutica de Zaragoza provincial (1894)

- Asociación Médico Farmacéutica del distrito de Sos (1894)

- Colegio Médico Farmacéutico de Baleares (1894)

- Colegio Médico Farmacéutico de Logroño (1894)*

- Asociación Médico Farmacéutica de Almunia de Doña Godina (1895)

- Instituto Nacional de Bacteriología e Higiene (1895)*

- Instituto Nacional de Terapéutica Operatoria (1895) 
PODER ARROYO MEDINA

- Instituto Nacional de Higiene Pública y Bacteriología (1896)*

- Asociación Médico Farmacéutica del distrito del Pilar (1896)

- Colegio de Farmacéuticos de Murcia (1897)*

- Ateneo Barcelonés (1899)

- Instituto Nacional de Bacteriología Alfonso XIII (1899)* 